\title{
RÉPLICA A JORGE MALEM: LAS CRÍTICAS QUE NO SE MERECE EL FEMINISMO RADICAL
}

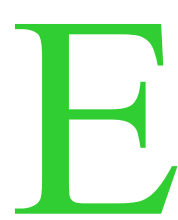

ntiendo que el artículo «Pornografía y Feminismo Radical» ${ }^{1}$, en el cual el profesor Jorge Malem presenta severas críticas contra el feminismo radical ${ }^{2}$, puede ser objeto, a su vez, de numerosas objeciones, aun cuando, como en mi caso, uno no se adhiera dogmáticamente al discurso de esta rama del feminismo ${ }^{3}$. Me parece que las objeciones que el profesor Malem presenta son en cierta medida excesivas, y en muchos casos injustas, respecto de una doctrina que influyó positivamente en la práctica (en todo lo que puede influirse desde el ámbito académico), para mejorar la suerte de un colectivo desaventajado, como lo es el de las mujeres. Mi réplica al profesor Malem abarca distintas cuestiones, algunas generales y otras más específicas, y a todas ellas intentaré referirme en los renglones que siguen.

Antes de detallar las prometidas objeciones, quisiera poner en claro, al menos a grandes rasgos, cuál es mi posición en cuanto al tema de fondo que aquí se trata. Entiendo que, en las intuiciones básicas, coincido con J. Malem en dos puntos fundamentales: a) Creo que un «buen» liberalismo pueda dar perfecta cuenta de muchos de los problemas que le interesan al F.R. (como trato de señalar en mi trabajo arriba mencionado). En este sentido, considero que el aluvión de críticas que el F.R. suele dirigir contra las posiciones más liberales es injustificable. b) Aunque simpatizo con la mayoría de las posiciones feministas y radicales, no llego a compartir la vocación censora del F.R. en materia de pornografía. De todos

${ }^{1}$ Revista «Doxa», núm. 12, 1992.

${ }^{2}$ Usaré como abreviatura de esta expresión, en algunos casos, las siglas F.R.

${ }^{3}$ Así, por ejemplo, en mi trabajo ¿Feminismo vs. Igualitarismo? Manuscrito. Universitat Pompeu Fabra, 1993. 
modos, y a pesar de estas centrales coincidencias con Malem, discrepo fundamentalmente con los argumentos que él presenta para sostener estas mismas ideas. Más aún, el trabajo de Malem deja entrever una postura de enfrentamiento al feminismo en general y al F.R. en particular que, me parece, va más allá de la cuestión específica de la pornografía.

Si (contrastando con lo que identifico como la postura de J. Malem) en este trabajo defiendo muy especialmente el núcleo del ideario feminista, ello obedece a diversas razones, entre las cuales puedo mencionar las siguientes. En primer lugar (y tal como procuraré señalar con algunos datos concretos), el F.R. trata de cuestiones que son de extrema gravedad, como el hecho de que buena parte de la población, simplemente por haber nacido bajo la categoría «mujer», se enfrenta a desventajas y dificultades que ninguna persona merece $^{4}$. Por otra parte, aunque otras doctrinas, también igualitarias, pueden tratar los problemas de los que trata el feminismo, lo cierto es que, por alguna razón (quizá porque muchos de los que tienen acceso a mejores posiciones académicas son hombres), tales problemas no se han (o, al menos, no se habían) tratado con habitualidad y seriedad. Además, el F.R. ha puesto el acento de su crítica (entiendo yo, adecuadamente) en la existencia de una injusta distribución de poder entre hombres y mujeres. Como alguna vez pudo darse en el caso de la discriminación racial: no basta con reafirmar las cualidades diferenciales de los negros, o permitirles acceder a derechos básicos como el voto, o una buena educación. Es importante, también, revertir toda una pesada carga de prejuicios, una red de discriminaciones que nadie explicita pero que cualquier persona razonable puede reconocer. Esta tarea, a la que se ha abocado especialmente el F.R., me parece encomiable y bien encaminada, más allá de muchas precisiones que en ocasiones me interesaría afirmar. Permítanme, ahora sí, concentrarme en el artículo del profesor Malem.

\section{Objeciones generales}

1. Crítica de la peor versión: En primer lugar, señalaría que una importante virtud de toda crítica, me parece, es la de presentar a la obra criticada en su mejor forma. En este sentido, creo que Jorge

${ }^{4}$ Esto se puede ejemplificar, aún en la mayoría de los países desarrollados, mostrando las enormes dificultades que, por el solo hecho de ser mujeres, las mujeres enfrentan (doy sólo un caso) al pretender «escalar posiciones» dentro de una cierta empresa. Decir esto, obviamente, no implica negar que los hombres puedan enfrentar (o de hecho enfrenten) otro tipo de problemas particulares. 
Malem ha tomado del feminismo radical sus peores párrafos; sus argumentos más débiles, y no los mejores; y esto es algo que se trasluce en todo el artículo de Malem. Alguno me podrá decir, es que el F.R. no tiene buenos argumentos. Pero esta es una afirmación que me parece, en principio, falsa (según intentaré demostrar). Malem podrá decir: es que ellas formulan, efectivamente, este tipo de afirmaciones exageradas. Sí, esto puede ser cierto, como lo es que, en nombre del liberalismo, se han sugerido y llevado a la práctica decisiones muy difícilmente aceptables. Pero no aceptaríamos que se critique al liberalismo por las aberraciones que se han escrito o hecho en su nombre. Creo que es posible, y además conveniente, reconstruir de otro modo el núcleo central de las ideas que queremos criticar 5 . Entiendo que hubiese sido preferible que J. Malem optase por este último camino en su crítica al F.R. ${ }^{6}$

${ }^{5}$ Anticipando una posible réplica a este trabajo, me interesaría responder brevemente a la pregunta: ¿se toma aquí la mejor versión del artículo de J. Malem? Al respecto diría, en primer lugar, que existe una gran diferencia entre criticar a un movimiento (al liberalismo, al conservadurismo, al feminismo, etc.) y criticar a un cierto autor. La primera tarea conlleva la necesidad de seleccionar autores, textos, épocas, etc., cosa que en el segundo caso aparece mucho más diluida. De allí que en la crítica a una cierta postura ideológica sea especialmente importante esto de «tomar la mejor versión». En la crítica a un autor, este riesgo aparece bastante más atenuado. Además, en este caso no estoy tomando en cuenta, ni siquiera, a un cierto autor, sino a un artículo específico de un cierto autor. El material a seleccionar, en este sentido, se encuentra infinitamente más acotado de lo que ocurre en la crítica a una corriente de pensamiento.

${ }^{6}$ Presentaré, a continuación, algunos casos en donde puede verse claramente que J. Malem ha tomado en cuenta una versión muy pobre del F.R. A) Sostener que para el F.R. las mujeres no pueden ser seres violentos (jamás), mientras que los hombres lo son siempre, resulta injusto respecto de la corriente que se critica, por más que Malem pueda encontrar alguna cita sugiriendo dicha idea (ver J. Malem, págs. 187, 188). Una reconstrucción adecuada, me parece, podría decir que las mujeres son normalmente objeto de violencia masculina, situación que a la inversa no suele presentarse. Baste citar, por ejemplo, algunas cifras que el F.R. normalmente presenta (y que intuitivamente me resulten razonables, aunque no conozca exactamente la confiabilidad de las mismas): el 44 por 100 de las mujeres norteamericanas sufrieron intentos de violación; el 85 por 100 acoso sexual en el trabajo: de $1 / 3$ a 1/4 de las mujeres casadas, violencia marital; el 70 por 100 de los crímenes de mujeres los provocan hombres, del mismo modo que casi todos los crímenes de hombres los cometen hombres, etc. (ver C. MacKinnon, Feminism Unmodified, Harvard U.P., 1987). B) Otro ejemplo: ni siquiera la muy radical C. MacKinnon está dispuesta a defender sin más la asimilación entre coito consensuado y violación, que Malem 
2. Las indeseables alianzas tácticas: En numerosas oportunidades (páginas 179, 208 ó 210, por ejemplo), Jorge Malem acusa al feminismo radical por las «alianzas tácticas» que acepta, en pos de algunas de sus causas. Típicamente, en el ámbito de la pornografía, dicha corriente aparece aliada a los sectores más conservadores de la sociedad, a la Iglesia, a los grupos ultratradicionalistas, etc. ¿Sería justo, sin embargo, criticar a Jorge Malem por el hecho de que, en su crítica al feminismo radical, él aparece aliado exactamente a tales sectores conservadores? ${ }^{7}$ Seguramente él estaría de acuerdo conmigo en que no ${ }^{8}$.

3. Generalizaciones y excepciones: Creo que, en el artículo en cuestión, Jorge Malem incurre numerosas veces en razonamientos falaces. Particularmente, Malem tiende a objetar al F.R. mostrando que muchos de los argumentos usados por tal corriente encuentran contraejemplos o excepciones en la realidad. Me parece claro, sin

parece generalizar a todas las feministas radicales, a partir de una cita de A. Carter (J. Malem, pág. 187). En general, lo que muy razonablemente quiere decir el F.R. es que, relaciones sexuales que se toman habitualmente como consensuadas aparecen, muchas veces, cargadas de violencia hacia la mujer. Más aun, los jueces, para evaluar si hubo en algún caso violación o no, tienden a ver si se produjo «mayor uso de la fuerza que el habitual», con lo cual presuponen lo que gente como MacKinnon razonablemente critican. Esto es, que muchas veces se fuerza a la mujer a tener relaciones sexuales cuando ella no lo desea. (Esto, obviamente, no implica afirmar que este tipo de relaciones forzadas ocurre «siempre», ni que no existen «mujeres violentas», al decir de J. Malem). Seguir los consejos delF.R., por ejemplo, llevaría a los jueces a ser más benignos en la evaluación de datos probatorios de violación, frente a una tarea de presentación de pruebas que normalmente resulta complicadísima (y emocionalmente demasiado costosa) para las mujeres. Ver, Catharine MacKinnon, Feminism... C) Como muchos críticos del F.R., Malem procura ridiculizar argumentos del feminismo, mostrándolos atados a una cierta (e inaceptable, sin dudas), «teoría conspirativa». (Sostiene Malem, en este sentido, que para el F.R. «la pornografía... está firmemente interesada en la dominación y subordinación de la mujer...»; o el «Estado democrático y su derecho está diseñado para acallar y subordinar a la mujer». J. Malem, pág. 196). Sin embargo, entiendo que los comportamientos que el F.R. critica son tan objetables en caso de ser intencionales, como lo serían si sólo constituyesen meros «by-products» sub-productos, comportamientos no explícitamente intencionales. Por ejemplo, puede que la mayoría de los hombres no tengan intenciones perversas cuando, directa o indirectamente, presionan a sus esposas para que se queden en «su natural ámbito del hogar», o se dediquen con exclusividad al «cuidado del esposo y de los críos» (podría tomar casos mucho más graves para ejemplificar esta situación de «natural subordinación»). De todos modos, aunque tales actitudes no resulten mal intencionadas, ellas pueden ser obviamente objetadas, como reproduciendo parámetros de conducta indeseables, desigualitarios, etc. Un liberal no convencionalista, como Jorge Malem, no debiera rechazar o pasar por alto esta observación.

${ }^{7}$ Que son los que habitualmente critican con más severidad al F.R.

${ }^{8}$ Adicionalmente, me interesaría aclarar (para evitar confusiones adicionales) que las mentadas «alianzas tácticas» entre feministas radicales y conservadores no deben verse como una «tendencia» del F.R. en su evolución política como movimiento. Simplemente hay una coincidencia de reclamos con el conservadurismo en algún punto, del mismo modo que hay una diferencia sustancial con tal tipo de posiciones en muchos otros aspectos. 
embargo, que aunque las excepciones sean numerosas, las generalizaciones pueden ser perfectamente bienvenidas, si es que nos ayudan a describir adecuadamente la realidad. Típicamente, Malem se pregunta, «¿acaso todos los hombres se comportan empíricamente de una manera violenta mientras ninguna mujer actúa de esa manera?» (pág. 189). La respuesta, qué duda cabe, es no. Sin embargo, acordar con Malem en esa respuesta, en nada favorece su argumentación sustantiva, porque lo importante es determinar si muchos hombres se comportan habitualmente de manera violenta frente a las mujeres. Del mismo modo, si alguien hubiese hablado del carácter racialmente discriminatorio de los norteamericanos, al menos hasta bien entrado este siglo, dicha generalización no podría haber sido adecuadamente descalificada, me parece, mostrando la benevolencia de algunos blancos hacia los negros o los éxitos profesionales de algunas personas de color. Lo importante, en este caso, era comprobar que la mayoría de los negros eran habitualmente discriminados. Tales generalizaciones sirven para hacernos más entendible la realidad y, como tales, merecen ser consideradas y aceptadas (en tanto no sean, claramente, malas descripciones, lo cual no parece ocurrir ni en el caso de la violencia racial, ni en el caso de la violencia sexual). Pretender que las feministas hablen de todos los casos, y de todas las circunstancias, cuando generalizan me resulta una mala reconstrucción de lo que el F.R. quiere decir ${ }^{9}$.

${ }^{9}$ Jorge Malem, sin embargo, utiliza con mucha frecuencia este tipo de críticas. Así puede verse en ejemplos como los siguientes, que me parecen todos ellos muy importantes: i) señala, para debilitar las propuestas antipornográficas de las feministas, que existen mujeres que consumen, y otras que producen, pornografia: ii) dice que las propuestas de A. Dworkin y C. MacKinnon demuestran que las mujeres no han sido acalladas (pág. 198); iii) sostiene que la existencia de pornografia homosexual, por caso, demuestra que la pornografía no se limita exclusivamente a degradar a la mujer (pág. 199); iv) afirma que una ordenanza antipornográfica difícilmente sea capaz de modificar las creencias de las mujeres (pág. 209). Yo le respondería. por ejemplo: i) que la presencia de mujeres en la producción o el consumo de pornografía no debilita en absoluto el argumento de las feministas, del mismo modo que la presencia del juez negro Clarence Thomas en la Corte Suprema norteamericana, defendiendo legislación racista, no le quita argumentos a la gente de color, para pronunciarse en contra de toda discriminación racial; ii) que el hecho de que Dworkin y MacKinnon puedan hablar no prueba demasiado respecto de las posibilidades reales de las mujeres para expresarse, como (permítaseme el ejemplo) los gritos de libertad de Espartaco no pueden servir para negar la existencia de esclavitud en la antigüedad; iii) que la existencia de pornografía homosexual no niega en absoluto que la pornografía más común pueda servir para degradar a la mujer (que, según entiendo. es el punto en cuestión); iv) que si una ordenanza no basta para tal fin (cambiar la mentalidad femenina), ello no demuestra tampoco que sea inútil, o poco importante, respecto de tal objetivo, en tanto contribuya adecuadamente al logro del mismo (más allá de la plausibilidad o no del objetivo mismo). 
4. La falsa conciencia: Según me parece, muchas veces, las feministas radicales utilizan inadecuadamente la idea de «falsa conciencia», de modo que quedan sólo ellas como conocedoras de las soluciones que las mujeres oprimidas necesitan. Sin embargo, no sé cuál es el lugar que, según Malem, debieran asumir los/las intelectuales, sino el de mirar críticamente a la realidad, y tratar de difundir sus opiniones, más allá de que no quede claro cómo instrumentar las propuestas concretas que realicen. En este sentido, Malem critica a las feministas dado que, a partir de las opiniones de éstas, no se sabría quiénes deberían diseñar una política a favor de las mujeres. Según las feministas -señala- dicha tarea no podría ser realizada ni por el Estado (descrito por el F.R. como represivo); ni por las mujeres (descritas por el F.R. como munidas de falsa conciencia). Con ironía, concluye Malem, según parece inferirse de lo dicho, los mismos hombres o, si no, las feministas radicales «esclarecidas», serían los únicos seres capacitados para llevar adelante tal política paternalista. Respuestas que, quiere sugerirnos Malem, parecen ambas contraintuitivas.

Frente a esta objeción, yo plantearía el siguiente ejemplo: ¿Cuál debería ser el papel, dentro de una sociedad donde reina la servidumbre, de un intelectual crítico? ¿Qué podría hacer, sino tratar de mostrarle a quienes aceptan sumisamente su propia servidumbre (como pudo ocurrir en la Edad Media) que dicha solicitud es inaceptable? ¿Debería criticarse a dicho intelectual por soberbia? ¿Debería exigírsele que exhiba soluciones concretas para los males que describe? Es claro, en este caso también, ni la autoridad reinante, ni los propios siervos (posiblemente), diseñarían una política «liberadora». No por ello, de todo modos, descalificaríamos la labor del intelectual crítico. Menos aún lo criticaríamos señalando que, según su propuesta, la nobleza o él mismo parecerían ser los únicos seres capacitados para reconstruir la sociedad.

5. Opresión económica y consenso: A través de su objeción a la idea de falsa conciencia, y otras afirmaciones aisladas (por caso, pág. 190), el profesor Malem parece suscribir algunas afirmaciones clásicas en el liberalismo conservador (lo cual no es en sí criticable, entiéndase bien). Por ejemplo, la de que no pueden objetarse aquellos «actos entre adultos que consienten ${ }^{10}$. Me parece que, sin embargo, un «buen liberal» (si es que se me permite usar esta expresión) debiera estar dispuesto a decir muchas cosas al respecto todavía.

${ }^{10}$ Ver, por ejemplo, R. Nozick, en su libro «Anarchy, State, and Utopia» (Oxford, 1974). 
Fundamentalmente, cualquier persona razonable, como Nozick o Malem, estaría dispuesta a aceptar que en el citado no habría (por definición) «consenso» si la persona en cuestión firmó un contrato (por ejemplo) bajo la amenaza de uso de fuerza por parte de otra, que le obligaba a firmar (éste sería el caso, según MacKinnon, de Linda Lovelace, y la película que protagonizó: «Garganta Profunda». J. Malem, pág. 181). Basta con aceptar este razonable supuesto, me parece, para advertir que la plausibilidad de usar la idea de consenso en la justificación de un cierto acto, depende del modo en que definamos la palabra «fuerza», o la palabra «consenso». Desde mi punto de vista (aunque acepto que esta afirmación resulta especialmente discutible), el liberalismo debiera estar dispuesto a cuestionar como no autónomos (tal como lo hace J. Raz) aquellos actos que se decidieron sin contar con «un adecuado abanico de opciones» a la hora de elegir ${ }^{11}$. Los casos difíciles, al respecto, serían muchos. Sin embargo, este criterio, desde mi óptica, nos ayudaría a reconstruir adecuadamente casos importantes: por ejemplo, no tomaríamos como autónomo el comportamiento de una mujer que no abandona su hogar, a pesar de sufrir violencia marital, si (como suele ocurrir) ella no cuenta con recursos económicos suficientes para dejar el hogar (ni tiene mayores esperanzas en las cuotas alimentarias que podría pasarle el golpeador); ni tiene resto suficiente (emocional, físico, etc.) para llevar adelante (y básicamente por sí sola) la educación de sus hijos; ni tiene el coraje necesario para admitir, frente a sus amigas, vecinas, familiares, que la han estado golpeando sistemáticamente. En situaciones como ésta, entiendo yo, no debiera decirse que la mujer «consiente» quedarse en su casa.

Situaciones como la anterior pueden verse perfectamente reproducidas en otros casos. Por ejemplo, entiendo que muchas sociedades modernas diseñan tal sistema de incentivos y desincentivos económicos que, para mucha gente, el margen de maniobra para ejercer su autonomía queda casi reducido a cero $^{12}$. Para tomar algunos casos

${ }^{11}$ Joseph Raz, The Morality of Freedom (Oxford U. P., 1989), pág. 373.

${ }^{12}$ Un liberal que criticó firmemente las propuestas del F.R. en materia de pornografía, como lo es R. Dworkin, reconoce también, en este sentido, que «Nuestro sistema económico... hace muchas veces difícil que las mujeres encuentren un trabajo satisfactorio, a través del cual puedan verse realizadas, y, por el contrario, muchas veces hace que ellas terminen aceptando roles en filmes pornográficos que de otro modo rechazarían». Este argumento que aporta Dworkin, obviamente, no me basta para probar que la elección de las mujeres, en casos como el citado, no es libre. Sí me sirve, en cambio, para mostrar que, muy razonablemente, un liberal puede reconocer el impacto negativo del sistema económico en la libertad de elección de alguien. Mi sugerencia va más allá, e implica, al menos, ser más cuidadoso al equiparar 
claros, pensemos en la prostitución en Centroamérica, la pornografía en Brasil, o la distribución de drogas en los Estados Unidos. En ambos casos, i) la situación de miseria de algunas personas es tan desesperante, y ii) los incentivos económicos para involucrarse en tareas «indeseables» (como la pornografía, la prostitución o la distribución de droga) tan altos, que tales personas se ven «coercionadas» (bajo la amenaza, diría, del hambre para sí y sus familiares) a aceptar tales tareas. Acepto que esta definición de coerción es muy amplia. De todos modos, con esto sólo me interesa recalcar que, también en este ámbito, las propuestas de las feministas radicales pueden tener muchos visos de razonabilidad.

6. Confusión entre feminismo radical y «feminismo de la diferencia»: La descripción según la cual la mujer es, por naturaleza, «tierna, abnegada, protectora, pacífica e igualitaria» (J. Malem, pág. 188) no debe ser identificada con el feminismo radical (que rechaza esta presentación), sino con el llamado «feminismo de la diferencia», corriente que tiende a ser ejemplificada con las posturas de Carol Gilligan ${ }^{13}$.

\section{Objeciones específicas}

Dentro de esta sección voy a incluir, básicamente, algunos puntos concretos en los que disiento con Jorge Malem, menos generales o (a mi criterio) menos graves que los señalados con anterioridad.

1. Algunas afirmaciones empíricas dudosas: En el texto, Malem realiza algunas afirmaciones empíricas de dudosa verosimilitud. Por ejemplo, que la pornografía «no tiene una presencia generalizada en nuestras sociedades» (pág. 201), hecho que puede ser refutado, al menos aquí en España, en cualquiera de la infinitud de locales de venta de revistas que están en la calle. Otro caso: Malem señala como un dato relevante para reconocer que las mujeres pueden ser sujetos activos del delito de violación, el hecho de que el código penal español recepta y penaliza este caso (pág. 188). Creo que esta figura bien podría existir aun si el delito en cuestión no se

estas «elecciones forzadas», con «elecciones libres». Ver Ronald Dworkin, «Women and Pornography» The New York Review of Books (octubre, 1993), vol. XL, n. 17.

${ }_{13}$ Así, por ejemplo, ver en C. MacKinnon, Feminism...: y Carol Gilligan, From a Different Voice (Cambridge, 1982). 
hubiese producido jamás (los datos adicionales que aporta tampoco resultan importantes) ${ }^{14}$.

2. Algunas argumentaciones muy débiles: A lo largo del artículo he encontrado algunas afirmaciones difíciles de comprender, a no ser que se trate de argumentaciones muy frágiles. Por ejemplo, Malem sostiene (pág. 187) que la afirmación «de que el hombre es un ser violento sólo puede ser entendida bajo el supuesto de que la mujer es un ser no violento», La verdad es que, al menos yo, puedo entender perfectamente (y aceptar en su contenido sustantivo) la idea de que el hombre es violento, sin abrir ningún juicio adicional acerca de la mujer. Del mismo modo, entendería que me dijeran que los hombres son violentos, y las mujeres también; o que los hombres son violentos, pero los pájaros no.

Incurriendo en una modalidad argumentativa que más arriba censuro (en el punto 4), Jorge Malem realiza la siguiente afirmación: «aun cuando se admita que un material determinado degrada a una mujer determinada, no se infiere lógicamente que ese material degrade al conjunto de todas las mujeres del planeta. Del mismo modo que la sustracción de un radio cassette de un automóvil no implica un ataque a todos los automovilistas propietarios de radio cassette. Habría en quien sostuviera una posición semejante una falacia en la composición» (pág. 201). Realmente no sé qué es lo que quiere decir Malem con esta idea. La verdad es que me parece que, de haber una falacia de composición en juego, ella es la falacia en la que J. Malem incurre ${ }^{15}$.

3. La carga emotiva: Es cierto que muchas radicales cargan sus textos de testimonios desgarradores y frases conmovedoras o altisonantes. Estos testimonios y frases no constituyen argumentos, es claro,

${ }^{14}$ Entiendo, de todos modos, que Malem no necesitaba apoyarse en datos tan débiles para «probar» algo obvio: que tanto la mujer como el hombre pueden actuar de modo violento. Como señalé con anterioridad, pensar que es central para el F.R. sostener lo contrario resulta por demás equivocado.

${ }^{15}$ ¿Hay alguien que se atreva a decir que todas las mujeres del planeta, sin excepciones, se ven atacadas por la pornografia? ¿Puede tomarse el recurso retórico de alguna feminista (en todo caso) como representante de lo que dicen todas ellas? Pero, más aún, ¿es que acaso importaría encontrar más ejemplos de personas que entienden que todas las mujeres se ven degradadas? Seguramente que no, dado que es otra la cuestión interesante, como el mismo Malem podría admitir. Por ejemplo, J. Malem defiende, sin abundar en detalles, que se prohíba a los niños el consumo de pornografía «con el fin de que no se dañen a sí mismos» (por ejemplo, pág. 208). ¿Se infiere (lógicamente) de su argumento que todos los niños del mundo van a verse dañados por tal consumo? ¿Importaría conocer el caso de algunos niños que no se vean dañados, o es que nos basta (aquí, y en todos los casos de paternalismo legítimo) solamente con saber que muchos de ellos pueden ser afectados? 
y eso debe tomarse en consideración desde un comienzo. Sin embargo, acotaría también que, en ocasiones, el presentar ejemplos muy fuertes, muy dramáticos, puede servir de buena introducción para luego pasar a desarrollar y discutir argumentos. En tanto el F.R. no se quede en la mera narración de historias emotivamente cargadas, la estrategia no me parece incorrecta, frente a un público muchas veces reacio a reconocer la importancia y gravedad de los problemas que el feminismo trata. Si, en cambio, el F.R. (como en ocasiones ocurre) ${ }^{16}$ se contentase con la mera exposición de anécdotas, y pretendiese con ello justificar ciertas decisiones, entonces, su posición resultaría muy debilitada. Por suerte, me parece, no es esta última una situación frecuente.

Lo señalado en este apartado vale tanto respecto del F.R., como respecto del mismo artículo de J. Malem, quien muchas veces utiliza frases cargadas emotivamente, como si se tratase de argumentos, o descripciones adecuadas de algunos hechos ${ }^{17}$.

4. El argumento de la «pendiente» o el «desliz»: Uno de los argumentos que Malem presenta, frente a la propuesta del F.R. de censurar, al menos, algún tipo de pornografía, es el conocido ejemplo de la «pendiente»: «La censura -señala- se sabe cuando comienza, pero no cuando termina» (pág. 203). Aunque, en líneas generales, es difícil rechazar esta idea, sólo motivado por un afán polemista diría que el mismo argumento de la «pendiente» está sujeto al argumento de la «pendiente». Con el argumento de la «pendiente», se sabe cuándo se comienza... Lo que me interesa decir es que, aunque normalmente existe un potencial efecto «desliz», «pendiente» o «cascada», ello no nos priva de tomar muchas medidas que vemos como aceptables. Por ejemplo, no vamos a dejar de penalizar algunos delitos por el riesgo de convertirnos en una sociedad represiva. Ese riesgo potencial, entendemos, puede ser conveniente y razonablemente limitado. Aún en la peligrosísima área de la libertad de expresión, puede muy razonablemente pensarse que ciertas proclamas incitando un levantamiento militar sean censuradas, sin tener que temer que, por ello, vamos a convertirnos en una sociedad censora ${ }^{18}$. Quiero decir, los riesgos potenciales no bastan para impedir razonables medidas de activismo estatal.

${ }^{16}$ Tal vez, por caso, en el libro Only Words, de C. MacKinnon (Harvard U.P., 1993).

${ }^{17}$ Aquí va algún ejemplo: Según Malem, no se puede aceptar que la pornografía «muestra a la mujer preocupada sólo por su goce sexual, tal como lo hacen, Susan Griffin y concordantemente con ella el Papa Carol Wojtyla» (pág. 192). Otro ejemplo: «el despreciable aparato conceptual utilizado» por el feminismo radical... (pág. 186).

${ }^{18}$ Acepto, sí, que si lo que tomamos en cuenta para evaluar los riesgos en juego es 
Espero que estas críticas puedan contribuir a enriquecer el debate en torno al F.R. $\mathrm{y}$, más fundamentalmente aun, a proteger a esta corriente de pensamiento frente a las objeciones que muchas veces, me parece, recibe de modo inmerecido.

la ordenanza antipornográfica que A. Dworkin y C. MacKinnon redactaron contra la pornografía las vaguedades son tantas que bien podríamos temer por la libertad de expresión. Sin embargo, al menos, no queda negada la posibilidad de redactar una ordenanza antipornográfica más acotada y precisa, si es que se encontrase que este objetivo es plausible. 
